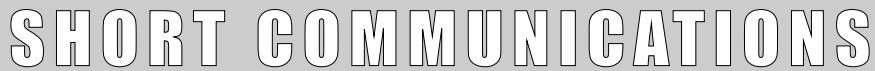

\section{Management of Toxoplasma gondii screening in pregnancy: light and shade}

\author{
Massimo De Paschale, Carlo Agrappi, Luisa Belvisi, Debora Cagnin, Teresa Cerulli, \\ Maria Teresa Manco, Laura Marinoni, Alessia Paganini, Pierangelo Clerici \\ U.O.C. Microbiologia \\ A.O. Ospedale Civile di Legnano (Mi)
}

Key words: Anti-Toxoplasma antibodies, Screening of Toxoplasma, Pregnancy

Gestione dello screening per infezione da Toxoplasma gondii in gravidanza: luci ed ombre

\section{SUMMARY}

The aim of our study was to evaluate the performance of screening for toxoplasmosis in pregnancy through comparing the data obtained from Italian and foreign women. 3074 women, 2465 Italians (80.2\%) and 609 foreigners (19.8\%) had undergone screening for serological detection of anti-Toxoplasma IgG and IgM antibodies in the years $2005-2007.85 .0 \%$ of the Italian women and $84.1 \%$ of the foreign women had the first blood test in the first trimester of pregnancy (difference not statistically significant). Among anti-Toxoplasma negative women average of blood tests during pregnancy was 3.69 for the Italians and 3.42 for the foreigners $(p<0.01) .32 .2 \%$ of Italian and $28.3 \%$ of foreign women were subjected to five or more blood tests (difference not statistically significant). Considering the trimester of pregnancy, the percentage of Italian and foreign women tested at least once in every trimester was respectively $58.0 \%$ and $45.2 \%(\mathrm{p}<0.01$ ). In conclusion, our data show that on the one hand the screening is active in controlling women within the first trimester of pregnancy, while on the other it appears weak in fully implementing the follow up, as indicated by the legislation currently in force. In addition, foreign women undergo fewer tests covering all trimesters compare to Italian women. This problem should be taken into account in terms of health policy.

\section{RIASSUNTO}

Scopo del nostro lavoro è stato quello di valutare l'andamento dello screening in gravidanza per la toxoplasmosi confrontando i dati ottenuti da donne di origine italiana e straniera. Sono state considerate 3074 donne, di cui 2465 italiane $(80.2 \%)$ e 609 straniere $(19.8 \%)$ nel triennio 2005-2007 che si erano sottoposte allo screening sierologico per la ricerca di anticorpi antiToxoplasma IgG e IgM. L'85\% delle donne italiane e 1'84.1\% delle donne straniere si sono presentate al primo controllo entro il I trimestre di gravidanza (differenza statisticamente non significativa). Tra le donne anti-Toxoplasma negative la media dei controlli eseguiti durante la gravidanza è stata di 3.69 per le donne italiane e 3.42 per le donne straniere $(\mathrm{p}<0.01)$. Il $32.2 \%$ delle italiane e il $28.3 \%$ delle straniere si sono sottoposte a cinque o più controlli (differenza statisticamente non significativa). Considerando i trimestri di gravidanza, le per- centuali di donne italiane e straniere che si sono sottoposte ad almeno un controllo per trimestre sono state rispettivamente del $58 \%$ e $45.2 \%$ $(p<0.01)$. In conclusione dai nostri dati risulta che da una parte lo screening risulta attivo nel controllare le donne entro il I trimestre di gravidanza, mentre dall'altra appare deficitario nel riuscire ad attuare completamente il follow up, come indicato dalla normativa attualmente in vigore. Inoltre le donne straniere si sottopongono ad un minor numero di controlli che coprono meno tutti i trimestri rispetto alle donne italiane. Di questo problema si dovrà tener conto a livello di politica sanitaria considerando che la proporzione di donne gravide di origine straniera è in costante aumento nel nostro Paese.

\section{INTRODUZIONE}

L'infezione da Toxoplasma gondii è una delle principali patologie responsabili di infezioni congenite con gravi danni al feto (18). In Europa

\section{Corresponding author: Massimo De Paschale}

U.O.C. Microbiologia - A.O. Ospedale Civile di Legnano - Via Candiani 2, 20025 Legnano (MI)

Tel.033। 449319 Fax 033। 449578 - E-mail: massimo.depaschale@ao-legnano.it 
è stato stimato che la toxoplasmosi congenita colpisce da 1 a 10 neonati su 10.000 (15). L'incidenza e la gravità dell'infezione varia al variare del trimestre in cui l'infezione è stata contratta. La frequenza di trasmissione, infatti, aumenta proporzionalmente con l'età gestazionale mentre diminuisce la gravità dell'infezione $(8,10,11)$. A livello preventivo sono state proposte campagne di screening in pazienti gravide, pregravide o nei neonati oltre a campagne di educazione sanitaria, ma l'attuazione di screening sierologici in gravidanza, in base anche ai dati sulla prevalenza dell'infezione nella popolazione, non è raccomandato in alcuni Paesi (Gran Bretagna, Olanda, Norvegia e USA) dove non lo si ritiene giustificato dal punto di vista costo-beneficio $(7,13,14,19)$, mentre viene attuato, con modalità diverse, in altri Paesi come Francia, Belgio, Svizzera, Germania e Austria $(2,3,16)$. In Italia, dove le prevalenze anticorpali riportate variano dal $21 \%$ al $48 \%(1,6,9$, $17,20,21)$, l'attuale normativa (G.U. $N^{\circ} 245 \mathrm{del}$ 20/10/98) comprende la sierologia per la toxoplasmosi tra gli esami previsti per il controllo in gravidanza e indica di effettuare il primo controllo entro la $13^{\text {a }}$ settimana e gli altri a distanza di 30-40 giorni uno dall'altro fino al parto per un totale di 5-7 controlli. Nonostante questo, però, non è scontato che l'attuazione dello screening avvenga nei termini corretti. Infatti, uno studio KAP (conoscenza, attitudine, pratica) svolto in Italia negli anni '90 indica che solo il $65 \%$ dei ginecologi interpellati ricontrollava periodicamente durante la gravidanza le gestanti risultate sieronegative al primo controllo (4). Inoltre in letteratura è riportato che sia nei Paesi dove lo screening è consigliato sia dove non lo è le percentuali di donne sieronegative che non completano il follow up durante la gravidanza è del 30-35\% $(5,12)$. Infine, l'aumento dell'immigrazione nel nostro Paese negli ultimi anni (9) ha comportato che sempre un maggior numero di donne extracomunitarie venga interessato dal programma di screening in gravidanza, ma la gestione di tale screening in donne di cultura e lingua diversa potrebbe risultare particolarmente problematico. Scopo del nostro lavoro è stato, quindi, quello di valutare la conduzione dello screening sierologico effettuato nel triennio 2005-2007 su donne gravide sia di origine italiana che straniera.

\section{MATERIALI E METODI}

Sono stati retrospettivamente considerati i dati riguardanti 3074 donne (età meda 31.5; range 1546 anni) che avevano iniziato e concluso il periodo di gravidanza nel triennio 2005-2007. In tale periodo le donne si erano sottoposte allo screening sierologico per la ricerca di anticorpi antiToxoplasma IgG e IgM (ETI-TOXOK-G-PLUS, ETI-TOXOK-M reverse PLUS; Dia Sorin, Saluggia, Italia).

Delle 3074 donne 2465 (80.2\%) erano di origine italiana (età media 32.2; range 15-46) e 609 $(19.8 \%)$ di origine straniera (età media: 28.3, range 15-44). I dati presi in considerazione sono stati: il trimestre di gravidanza in cui è avvenuto il primo controllo, il numero e la media dei controlli effettuati durante la gravidanza e la distribuzione dei controlli in relazione ai vari trimestri.

Per l'analisi statistica sono stati usati il test chi quadro e il test esatto di Fisher per il confronto tra percentuali e il test $t$ di Student per il confronto tra medie.

\section{RISULTATI}

Entro il I trimestre di gravidanza si erano presentate per il primo controllo 2608 donne $(84.8 \%)$, mentre $302(9.8 \%)$ e $164(5.3 \%)$ si erano presentate per il primo controllo rispettivamente al II e al III trimestre (tabella 1). I dati, stratificati in base all'origine italiana o straniera delle donne sono riportati tabella 1. Le differenze tra donne italiane e straniere non sono statisticamente significative.

Al primo controllo:

- 639 donne (20.8\%) erano risultate antiToxoplasma IgG positive e IgM negative: in particolare $437(17.7 \%)$ donne italiane e $202(33.2 \%)$ donne straniere $(\mathrm{p}<0.01)$ (tabella 2$)$. La media dei controlli eseguiti in questo gruppo durante la gravidanza era stata di 1.25 (range 1-7): 1.23 (range 1-7) per le donne italiane e 1.29 (range 1-6) per le donne straniere (differenza statisticamente non significativa) (tabella 3). Di queste donne, 113 (17.7\%) avevano effettuato più di un controllo: 101 (89.4\%) avevano un risultato di antiToxoplasma IgG forte e 12 (10.6\%) debole.

- 53 donne (1.7\%) erano risultate anti-Toxoplasma IgM positive o dubbie (tabella 2 ) ed erano state sottoposte ad ulteriori accertamenti e, in caso di sospetta infezione acuta o recente, inviate a un Centro di Riferimento.

- 2382 donne erano risultate anti-Toxoplasma IgG e IgM negative $(77.5 \%)$. La media dei controlli era stata di 3.65 (range 1-9): 3.69 (range 1-9) per le donne italiane e 3.42 (range 1-8) per le donne straniere $(\mathrm{p}<0.01)$ (tabella 3$)$. Per quanto riguarda il numero di controlli in questo gruppo, 1993 donne $(83.7 \%)$ si erano sottoposte a più di un controllo: 1672 (84.2\%) donne italiane e $321(81.1 \%)$ donne straniere (differenza statisticamente non significativa) (tabella 4). In particolare 751 $(31.5 \%)$ donne si erano sottoposte a cinque o più 
controlli come indicato dall'attuale decreto ministeriale: $639(32.2 \%)$ donne italiane e $112(28.3 \%)$ donne straniere (differenza statisticamente non significativa) (tabella 4).

Considerando i trimestri di gravidanza, 1330 $(55.8 \%)$ donne sieronegative si erano sottoposte ad almeno un controllo per trimestre: 1151 (58.0\%) donne italiane e $179(45.2 \%)$ donne straniere $(\mathrm{p}<0.01)$ (tabella 5).

I dati relativi al numero e alla media dei prelievi in relazione ai trimestri di gravidanza stratificati per classi d'età sono riportati in tabella 6 .

Tabella I. Trimestre di gravidanza in cui è avvenuto il primo controllo dello screening per la presenza di anticorpi anti-Toxoplasma in donne italiane e straniere (triennio 2005-2007).

\begin{tabular}{|l|l|l|l|l|l|l|l|}
\hline & \multicolumn{2}{|c|}{ ITALIANE } & \multicolumn{2}{c|}{ STRANIERE } & & \multicolumn{2}{c|}{ TOTALE } \\
\hline $\begin{array}{l}\text { Trimestre } \\
\text { gravidanza }\end{array}$ & $\mathrm{N}^{\circ}$ & $\%$ & $\mathrm{~N}^{\circ}$ & $\%$ & $\mathrm{P}$ & $\mathrm{N}^{\circ}$ & $\%$ \\
\hline I trimestre & 2096 & 85.0 & 512 & 84.1 & $\mathrm{NS}$ & 2608 & 84.4 \\
\hline II trimestre & 240 & 9.7 & 62 & 10.2 & $\mathrm{NS}$ & 302 & 9.8 \\
\hline III trimestre & 129 & 5.2 & 35 & 5.7 & $\mathrm{NS}$ & 164 & 5.3 \\
\hline Totale & 2465 & & 609 & & & 3074 & \\
\hline
\end{tabular}

Tabella 2. Prevalenza degli anticorpi anti-Toxoplasma in gravide italiane e straniere (triennio 2005-2007).

\begin{tabular}{|l|l|l|l|l|l|l|l|}
\hline & \multicolumn{2}{|c|}{ ITALIANE } & \multicolumn{2}{c|}{ STRANIERE } & & \multicolumn{2}{c|}{ TOTALE } \\
\hline Anti-Toxoplasma & $\mathrm{N}^{\circ}$ & $\%$ & $\mathrm{~N}^{\circ}$ & $\%$ & $\mathrm{P}$ & $\mathrm{N}^{\circ}$ & $\%$ \\
\hline IgG neg/IgM neg & 1986 & 80.6 & 396 & 65.0 & $<0.01$ & 2382 & 77.5 \\
\hline IgG POS/IgM neg & 437 & 17.7 & 202 & 33.2 & $<0.01$ & 639 & 20.8 \\
\hline IgM POS (o dubbi) & 42 & 1.7 & 11 & 1.8 & $\mathrm{NS}$ & 53 & 1.7 \\
\hline Totale & 2465 & & 609 & & & 3074 & \\
\hline
\end{tabular}

Tabella 3. Media dei controlli eseguiti in gravidanza in relazione alla presenza di anticorpi anti-Toxoplasma in donne gravide italiane e straniere (triennio 2005-2007).

\begin{tabular}{|l|l|l|l|l|l|l|l|l|l|l|}
\hline & \multicolumn{3}{|c|}{ ITALIANE } & \multicolumn{3}{c|}{ STRANIERE } & \multicolumn{3}{c|}{ TOTALE } \\
\hline Anti-Toxoplasma & $\begin{array}{l}\mathrm{N}^{\circ} \\
\text { controlli }\end{array}$ & media & range & $\begin{array}{l}\mathrm{N}^{\circ} \\
\text { controlli }\end{array}$ & media & range & $\mathrm{P}$ & $\begin{array}{l}\mathrm{N}^{\circ} \\
\text { controlli }\end{array}$ & media & range \\
\hline $\begin{array}{l}\text { IgG neg/ } \\
\text { IgM neg }\end{array}$ & 7337 & 3.69 & $1-9$ & 1353 & 3.42 & $1-8$ & $<0.01$ & 8690 & 3.65 & $1-9$ \\
\hline $\begin{array}{l}\text { IgG POS/ } \\
\text { IgM neg }\end{array}$ & 535 & 1.23 & $1-7$ & 260 & 1.29 & $1-6$ & $\mathrm{NS}$ & 795 & 1.25 & $1-7$ \\
\hline $\begin{array}{l}\text { IgM POS } \\
\text { (o dubbi) }\end{array}$ & 100 & 2.38 & $1-6$ & 33 & 3.00 & $1-6$ & $\mathrm{NS}$ & 133 & 2.51 & $1-6$ \\
\hline Totale & 7972 & 3.24 & $1-9$ & 1646 & 2.70 & $1-8$ & $<0.01$ & 9618 & 3.13 & $1-9$ \\
\hline
\end{tabular}

Tabella 4. Numero dei controlli eseguiti in gravidanza in donne italiane e straniere risultate anti-Toxoplasma negative al primo controllo (triennio 2005-2007).

\begin{tabular}{|l|l|l|l|l|l|l|l|}
\hline & \multicolumn{2}{|c|}{ ITALIANE } & \multicolumn{2}{c|}{ STRANIERE } & & \multicolumn{2}{c|}{ TOTALE } \\
\hline $\mathrm{N}^{\circ}$ Controlli & $\mathrm{N}^{\circ}$ & $\%$ & $\mathrm{~N}^{\circ}$ & $\%$ & $\mathrm{P}$ & $\mathrm{N}^{\circ}$ & $\%$ \\
\hline 1 & 314 & 15.8 & 75 & 18.9 & $\mathrm{NS}$ & 389 & 16.3 \\
\hline$>1$ & 1672 & 84.2 & 321 & 81.1 & $\mathrm{NS}$ & 1993 & 83.7 \\
\hline Totale & 1986 & & 396 & & & 2382 & \\
\hline & & & & & & & \\
\hline$<5$ & 1347 & 67.8 & 284 & 71.7 & $\mathrm{NS}$ & 1631 & 68.5 \\
\hline$>=5$ & 639 & 32.2 & 112 & 28.3 & $\mathrm{NS}$ & 751 & 31.5 \\
\hline Totale & 1986 & & 396 & & & 2382 & \\
\hline
\end{tabular}


Tabella 5. Controlli eseguiti in relazione al trimestre di gravidanza in donne italiane e straniere nel triennio 2005-2007.

\begin{tabular}{|l|c|c|c|c|c|c|c|}
\hline & \multicolumn{2}{|c|}{ ITALIANE } & \multicolumn{2}{c|}{ STRANIERE } & \multicolumn{2}{c|}{ TOTALE } \\
\hline Controlli & $\mathrm{N}^{\circ}$ & $\%$ & $\mathrm{~N}^{\circ}$ & $\%$ & $\mathrm{P}$ & $\mathrm{N}^{\circ}$ & \multicolumn{2}{c|}{} \\
\hline Solo I trimestre & 204 & 10.3 & 54 & 13.6 & $<0.05$ & 258 & 10.8 \\
\hline $\begin{array}{l}\text { I trimestre + } \\
\text { II trimestre }\end{array}$ & 211 & 10.6 & 61 & 15.4 & $<0.01$ & 272 & 11.4 \\
\hline $\begin{array}{l}\text { I trimestre + } \\
\begin{array}{l}\text { II trimestre + } \\
\text { III trimestre }\end{array}\end{array}$ & 1151 & 58.0 & 179 & 45.2 & $<0.01$ & 1330 & 55.8 \\
\hline $\begin{array}{l}\text { I trimestre + II } \\
\text { trimestre }\end{array}$ & 81 & 4.1 & 36 & 9.1 & $<0.01$ & 117 & 4.9 \\
\hline Solo II trimestre & 81 & 4.1 & 17 & 4.3 & $\mathrm{NS}$ & 98 & 4.1 \\
\hline $\begin{array}{l}\text { II trimestre+ } \\
\text { III trimestre }\end{array}$ & 142 & 7.1 & 25 & 6.3 & $\mathrm{NS}$ & 167 & 7.0 \\
\hline Solo III trimestre & 116 & 5.8 & 24 & 6.1 & $\mathrm{NS}$ & 140 & 5.9 \\
\hline Totale & 1986 & & 396 & & & 2382 & \\
\hline
\end{tabular}

Tabella 6. Media di controlli eseguiti in gravidanza e numero di donne che si erano sottoposte ad almeno un controllo in ogni trimestre in relazione alle classi d'età (triennio 2005-2007).

\begin{tabular}{|c|c|c|c|c|c|c|c|c|c|c|}
\hline \multirow{3}{*}{ Prelievi } & \multicolumn{8}{|c|}{ CLASSI D'ETÀ } & \multirow{2}{*}{\multicolumn{2}{|c|}{ TOTALE }} \\
\hline & \multicolumn{2}{|c|}{$\leq 20$ anni } & \multicolumn{2}{|c|}{ 21-30 anni } & \multicolumn{2}{|c|}{$31-40$ anni } & \multicolumn{2}{|c|}{$>40$ anni } & & \\
\hline & media & range & media & range & media & range & media & range & media & range \\
\hline Italiane & 3.83 & $1-7$ & 3.69 & $1-9$ & 3.71 & $1-9$ & 3.28 & $1-8$ & 3.69 & $1-9$ \\
\hline Straniere & 3.84 & $1-7$ & 3.5 & $1-8$ & 3.26 & $1-7$ & 3.80 & $1-7$ & 3.42 & $1-8$ \\
\hline $\mathrm{P}$ & NS & & NS & & $<0.01$ & & NS & & $<0.01$ & \\
\hline $\begin{array}{l}\text { Controlli al } \\
\mathrm{I}+\mathrm{II}+\mathrm{III} \text { trimestre }\end{array}$ & $\mathrm{N}^{\circ}$ & $\%$ & $\mathrm{~N}^{\circ}$ & $\%$ & $\mathrm{~N}^{\circ}$ & $\%$ & $\mathrm{~N}^{\circ}$ & $\%$ & $\mathrm{~N}^{\circ}$ & $\%$ \\
\hline Italiane & $22 / 36$ & 61.1 & $372 / 649$ & 57.3 & $729 / 1248$ & 58.4 & $28 / 53$ & 52.8 & $1151 / 1986$ & 58.0 \\
\hline Straniere & $12 / 32$ & 37.5 & $110 / 234$ & 47.0 & $55 / 125$ & 44.0 & $2 / 5$ & 40.0 & $179 / 396$ & 45.2 \\
\hline $\mathrm{P}$ & NS & & $<0.01$ & & $<0.01$ & & NS & & $<0.01$ & \\
\hline
\end{tabular}

\section{DISCUSSIONE}

La gravità dell'infezione congenita da Toxoplasma gondii ha spinto alcuni Paesi ad attuare campagne di screening anticorpali per individuare possibili infezioni acute con rischio di trasmissione dell'infezione al feto. In Italia l'attuale normativa indica un protocollo di accesso agli esami di laboratorio comprendente la ricerca di anticorpi anti-Toxoplasma all'inizio della gravidanza, possibilmente entro la $13^{\mathrm{a}}$ settimana, e in caso di negatività per le IgG la ripetizione dell'esame ogni 30-40 giorni fino al parto per un totale di 5-7 controlli. Perché tale protocollo sia efficace è richiesta la piena collaborazione sia del medico prescrittore che della paziente oltre ad una efficiente organizzazione delle strutture sanitarie in grado di permettere l'accesso ai prelievi e la risposta dei risultati nei tempi richiesti. La verifica della conduzione di tale protocollo è di primaria importanza per poter valutare l'efficacia dello screening, comprendere i punti deboli e, quindi, pianificare programmi d'interventi mirati.

Nel nostro studio la stragrande maggioranza delle donne comincia il controllo entro il I trimestre di gravidanza come indicato dal decreto ministeriale. Ugualmente la stragrande maggioranza delle donne sieronegative effettua più di un controllo durante la gravidanza, ma solo un terzo circa esegue un numero di controlli (dai 5 ai 7) indicati dal decreto ministeriale. Inoltre, se si considerano i trimestri di gravidanza, solo poco più della metà effettua almeno un controllo per trimestre.

Per quanto riguarda la gestione dello screening tra le donne sieropositive in circa un quinto viene effettuato uno o più controlli aggiuntivi. Solo però in una piccola quota di queste persone la presenza di anticorpi anti-Toxoplasma IgG a basso titolo giustificherebbe un follow up, mentre per le altre la ripetizione dei controlli risulta inutile.

Se consideriamo le donne di origine straniera che sono circa un quinto del totale, mentre la conduzione dello screening è uguale a quella delle italiane riguardo al periodo del primo controllo, al contrario la media totale dei controlli e la percentuale di donne che li effettuano in ogni trimestre è statisticamente più bassa rispetto alle italiane.

Tale situazione può essere dovuta a diverse cause. Poiché l'età media delle donne straniere è più 
bassa rispetto a quella delle italiane, potrebbe esserci un bias dovuto all'età, ipotizzando una compliance differente per donne di età diversa. La stratificazione dei dati considerando le varie fasce d'età, tuttavia, pur riducendo le differenze, ha confermato la situazione. Si possono, quindi, ipotizzare altri fattori che possono spaziare dalle differenze culturali, linguistiche e sociali a problemi di integrazione riferibili all'epoca più o meno recente di immigrazione. Per poter approfondire questi aspetti andrebbero condotti appositi studi mirati. Inoltre nel nostro studio sono state considerate solo le donne che hanno avuto accesso al Servizio Sanitario Nazionale e non abbiamo indagato ciò che succede tra le donne immigrate clandestinamente che sfuggono alle normali indagini di screening.

\section{CONCLUSIONI}

Dai nostri dati risulta che la gestione dello screening in gravidanza presenta nel suo complesso luci e ombre. Da una parte lo screening è attivo nel reclutare le donne entro il I trimestre di gravidanza quando è più facile valutare e gestire una possibile infezione acuta. D'altra parte appare deficitaria l'attuazione completa dello screening come indicato dal decreto ministeriale attualmente in vigore. Inoltre la gestione nelle donne di origine straniera appare particolarmente problematica dal momento che si sottopongono ad un minor numero di controlli che non coprono tutti i trimestri in confronto alle donne italiane. L'entità del problema non è indifferente dal momento che la proporzione di donne gravide di origine straniera è in costante aumento nel nostro Paese e di questo si dovrà tener conto a livello di politica sanitaria

\section{RINGRAZIAMENTI}

Si ringrazia il Sig. Luigi Bertinotti per l'estrazione informatica dei dati inerenti alle donne in gravidanza.

\section{BIBLIOGRAFIA}

1. Adorisio E, De Cicco AL, Salandri A, Simili M, Annichiarico LS. Prevalence of Toxoplasma gondii infections in groups of individuals in Rome and its environment. Clin Ter 1996; 147: 317-20.

2. Agence Nationale d'Accréditation et d'Evaluation en Santé. La surveillance biologique de la femme enceinte en bonne santé et sans antécédents pathologiques. Paris: ANAES 1996.

3. Aspöck H, Pollak A. Prevention of prenatal toxoplasmosis by serological screening of pregnant women in Austria. Scand J Infect Dis 1992; 84 (suppl): 32-7.

4. Buffolano W, Gilbert RE, Holland FJ, Fratta D, Palumbo F, Ades AE. Risk factors for recent
Toxoplasma infection in pregnant women in Naples. Epidemiol Infect 1996; 116: 347-51.

5. Charrier L, Moiraghi Ruggenini A, Renga G, Ditommaso S, Lievre MA, Zotti C. Cost analysis of Toxoplasma infection control in pregnancy. Ann Ig 2003; 15: 505-14.

6. Condorelli F, Scalia G, Stivala A, et al. Seroprevalence to some TORCH agents in a Sicilian female population of fertile age. Eur J Epidemiol 1993; 9: 341-3.

7. Conyn van Spoondonek MAE. Prevention of congenital toxoplasmosis in The Netherlands. Bilthoven: National Institute of Public Health and Enviromental Protection. 1991 ISBN 90-90004179-6.

8. Daffos F, Forestier F, Capella-Pavlovsky M, et al. Prenatal management of 746 pregnancies at risk for congenital toxoplasmosis. $N$ Engl J Med 1988; 318: 271-5.

9. De Paschale M, Agrappi C, Clerici P, et al. Prevalence of Toxoplasma gondii infection in Italian and foreign female population living in the area of Legnano (Milan). Microbiol Med 2006; 21: 322-7.

10. Desmonts G, Couvreur J. Congenital toxoplasmosis. Prospective study of the outcome of pregnancy in 542 women with toxoplasmosis acquired during pregnancy. Ann Pediatr (Paris) 1984; 31: 805-9.

11. Dunn D, Wallon M, Peyron F, Petersen E, Peckham C, Gilbert R. Mother-to-child transmission of toxoplasmosis: risk estimates for clinical counselling. Lancet 1999; 353: 1829-33.

12. Eskild A, Fallås Dahl F, Melby KK, Nesheim BI. Testing for toxoplasmosis in pregnancy: a study of the routines in primary antenatal care. JMed Screen 2003; 10: $172-5$.

13. Eskild A, Oxman A, Magnus P, Bjørndal A, Bakketeiq LS. Screening for toxoplasmosis in pregnancy: what is the evidence of reducing a health problem? J Med Screen 1996; 3: 188-94.

14. Gilbert RE, Peckham CS. Congenital toxoplasmosis in the United Kingdom: to screen or not to screen? $J$ Med Screen 2002; 9: 135-41.

15. Gilbert RE. Epidemiology of infection in pregnant women. In: Petersen E, Amboise-Thomas P Eds. Congenital toxoplasmosis: scientific background, clinical management and control, Springer-Verlag, Paris (France) 2000.

16. Hengst P. Screening for toxoplasmosis in pregnant women: presentation of a screening programme in the former "East"-Germany and the present status in Germany. Scand J Infect Dis 1992; 84 (suppl): 38-42.

17. Moschen ME, Stroffolini T, Arista S, et al. Prevalence of Toxoplasma gondii antibodies among children and teenagers in Italy. Microbiologica 1991; 14: 229-34.

18. Remington JS, McLeod R, Desmonts G. Toxoplasmosis. In: Infectious diseases of the foetus and newborn infant, Remington JS, Klein JO Eds, Fourth Edition. WB Saunders, Philadelphia 1995; 140-243.

19. Royal College of Obstetricians and Gynaecologists. Antenatal care. Routine care for the healthy pregnant woman. London: RCOG 2003.

20. Valcavi PP, Natali A, Soliani L, Montali S, Dettori G, Chezzi C. Prevalence of anti-Toxoplasma gondii antibodies in the population of the area of Parma (Italy). Eur J Epidemiol 1995; 11: 333-7.

21. Vezzo R, Vigorè L, Goglio A, et al. Sieroprevalenza per anticorpi anti-Toxoplasma gondii in 14186 soggetti. Microbiol Med 2001; 16: 220. 